\title{
Transcriptome response to alkane biofuels in Saccharomyces cerevisiae: identification of efflux pumps involved in alkane tolerance
}

\author{
Hua Ling, Binbin Chen, Aram Kang, Jong-Min Lee and Matthew Wook Chang*
}

\begin{abstract}
Background: Hydrocarbon alkanes have been recently considered as important next-generation biofuels because microbial production of alkane biofuels was demonstrated. However, the toxicity of alkanes to microbial hosts can possibly be a bottleneck for high productivity of alkane biofuels. To tackle this toxicity issue, it is essential to understand molecular mechanisms of interactions between alkanes and microbial hosts, and to harness these mechanisms to develop microbial host strains with improved tolerance against alkanes. In this study, we aimed to improve the tolerance of Saccharomyces cerevisiae, a model eukaryotic host of industrial significance, to alkane biofuels by exploiting cellular mechanisms underlying alkane response.

Results: To this end, we first confirmed that nonane (C9), decane (C10), and undecane (C11) were significantly toxic and accumulated in S. cerevisiae. Transcriptome analyses suggested that C9 and C10 induced a range of cellular mechanisms such as efflux pumps, membrane modification, radical detoxification, and energy supply. Since efflux pumps could possibly aid in alkane secretion, thereby reducing the cytotoxicity, we formed the hypothesis that those induced efflux pumps could contribute to alkane export and tolerance. In support of this hypothesis, we demonstrated the roles of the efflux pumps Snq2p and Pdr5p in reducing intracellular levels of C10 and C11, as well as enhancing tolerance levels against C10 and C11. This result provided the evidence that Snq2p and Pdr5p were associated with alkane export and tolerance in S. cerevisiae.
\end{abstract}

Conclusions: Here, we investigated the cellular mechanisms of $\mathrm{S}$. cerevisiae response to alkane biofuels at a systems level through transcriptome analyses. Based on these mechanisms, we identified efflux pumps involved in alkane export and tolerance in S. cerevisiae. We believe that the results here provide valuable insights into designing microbial engineering strategies to improve cellular tolerance for highly efficient alkane biofuel production.

Keywords: Saccharomyces cerevisiae, Alkanes, Biofuels, Tolerance, Transcriptome, Efflux pumps

\section{Background}

Next-generation biofuels, such as long-chain alcohols, and fatty-acid- and isoprenoid-derived fuels, offer advantages of high energy density, low freezing point, and compatibility with the existing fuel storage and distribution infrastructure [1-4]. Recently, hydrocarbon alkanes, main components of fossil fuels, have been considered as important next-generation biofuels because their microbial production was demonstrated. Schirmer et al. converted intermediates of fatty acid metabolism to alkanes in

\footnotetext{
* Correspondence: Matthewchang@ntu.edu.sg

School of Chemical and Biomedical Engineering, Nanyang Technological University, 62 Nanyang Drive, Nanyang 637459, Singapore
}

Escherichia coli by introducing two enzymes involved in an alkane biosynthesis pathway from cyanobacteria [5]. Bernard et al. identified Arabidopsis alkane synthesis enzymatic components and reconstituted plant alkane biosynthesis in yeast [6].

Despite these successes in microbial alkane production, the toxicity of alkanes to microbial hosts can eventually be a bottleneck for high productivity. To overcome this toxicity issue, it is imperative to develop engineering strategies to improve microbial tolerance against biofuel alkanes, which requires a clear understanding of molecular mechanisms of interaction between microbial hosts and alkanes. Physiologically, hydrocarbon accumulation in cell membrane causes loss of membrane integrity and function, which ultimately

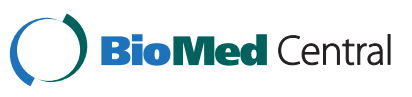


leads to cell death [7]. In response, cells protect themselves against the toxicity of hydrocarbons by ordering the lipid bilayer to modify lipopolysaccharide (LPS) and cell wall/Slayer hydrophobicity, as well as activating the excretion by energy-consuming transport systems [8]. Despite the aforementioned studies, which primarily focused on physiological or cytological effects, there is lack of understanding of the molecular mechanisms of interactions between microbes and alkanes especially at a systems level.

Toxicogenomics, which combines genomics and toxicology, is useful for identification of toxicants and their putative mechanisms of action at a systems level. Recently, toxicogenomics has been applied to elucidate mechanisms underlying environment stresses and chemical toxicity to microorganisms $[9,10]$. For instance, Chang et al. studied the toxicogenomic response of pathogens to antimicrobials by using microarray-based transcriptome analyses [11]. Carvalho et al. studied transcriptomic response in marine diatom Thalassiosira pseudonana exposed to benzo[a]pyrene [12]. Notably, toxicogenomics has also offered an effective means to study the molecular mechanisms of cell response to organic solvents. For example, based on genome-wide microarray analyses, our group previously identified and reconstituted genetic regulatory networks to improve the tolerance of E. coli against isooctane [13]. In Saccharomyces cerevisiae strain KK-211, genes involved in tolerance to organic solvents were successfully identified based on transcriptome analyses $[14,15]$. Note that $S$. cerevisiae, an established and widely used eukaryotic model for molecular and cellular biology studies, has been used as an experimental model in toxicogenomics [16-18]. In this light, this study aimed to (i) investigate molecular mechanisms underlying cellular response to alkanes at the systems level and (ii) improve cellular tolerance based on these mechanisms in S. cerevisiae, a model eukaryotic host of industrial significance. Briefly, we showed that nonane (C9) and decane (C10) induced cellular mechanisms associated with efflux pumps, membrane modification, radical detoxification, and energy supply. More importantly, based on these cellular mechanisms, we demonstrated that efflux pumps Snq2p and Pdr5p played roles in reducing intracellular levels of $\mathrm{C} 10$ and undecane (C11), and enhancing tolerance levels against $\mathrm{C} 10$ and $\mathrm{C} 11$. Given the aforementioned recent reports on microbial alkane production $[5,19]$, we envision that the engineering strategy established in this study can readily be extended to develop robust microbial hosts for alkane biofuels production.

\section{Results and discussion}

Toxicity and intracellular accumulation of alkanes

We first examined the toxicity of nonane (C9), decane (C10), undecane (C11), and dodecane (C12). In this work, we primarily focused on $\mathrm{C} 9-\mathrm{C} 12$ because our preliminary studies had indicated low cytotoxicity of $\mathrm{C} 13$ and longer alkanes. S. cerevisiae BY4741 cells were incubated in the medium containing each alkane $(2 \%[\mathrm{v} / \mathrm{v}])$. Figure 1A shows that cell viability was decreased upon exposure to $\mathrm{C} 9-\mathrm{C} 12$. In particular, treatment with C9C11 decreased cell viability by about $50 \%$. Based on this result, we hypothesized that $\mathrm{C} 9-\mathrm{C} 12$ might affect cell membrane permeability or integrity. Figure $1 \mathrm{~B}$ shows that the intensity of red fluorescent signal of propidium iodide (PI) and the ratio of the fluorescent signals (PI/ SYTO9) were increased upon exposure to C9-C12. If more PI molecules penetrate into cell wall/membrane and stain nucleic acids, PI intensities and PI/SYTO 9 ratios become higher. Therefore, this result suggests that C9-C11 increased cell membrane permeability. Among these alkanes, C9 and C10 resulted in the highest PI intensities and PI/SYTO9 ratios, which indicates the highest membrane permeability. To determine whether this increased membrane permeability was contributed by damage on cell surface, cell surface was examined using Field Emission Scanning Electron Microscopy (FESEM). As shown in Additional file 1: Figure S1,

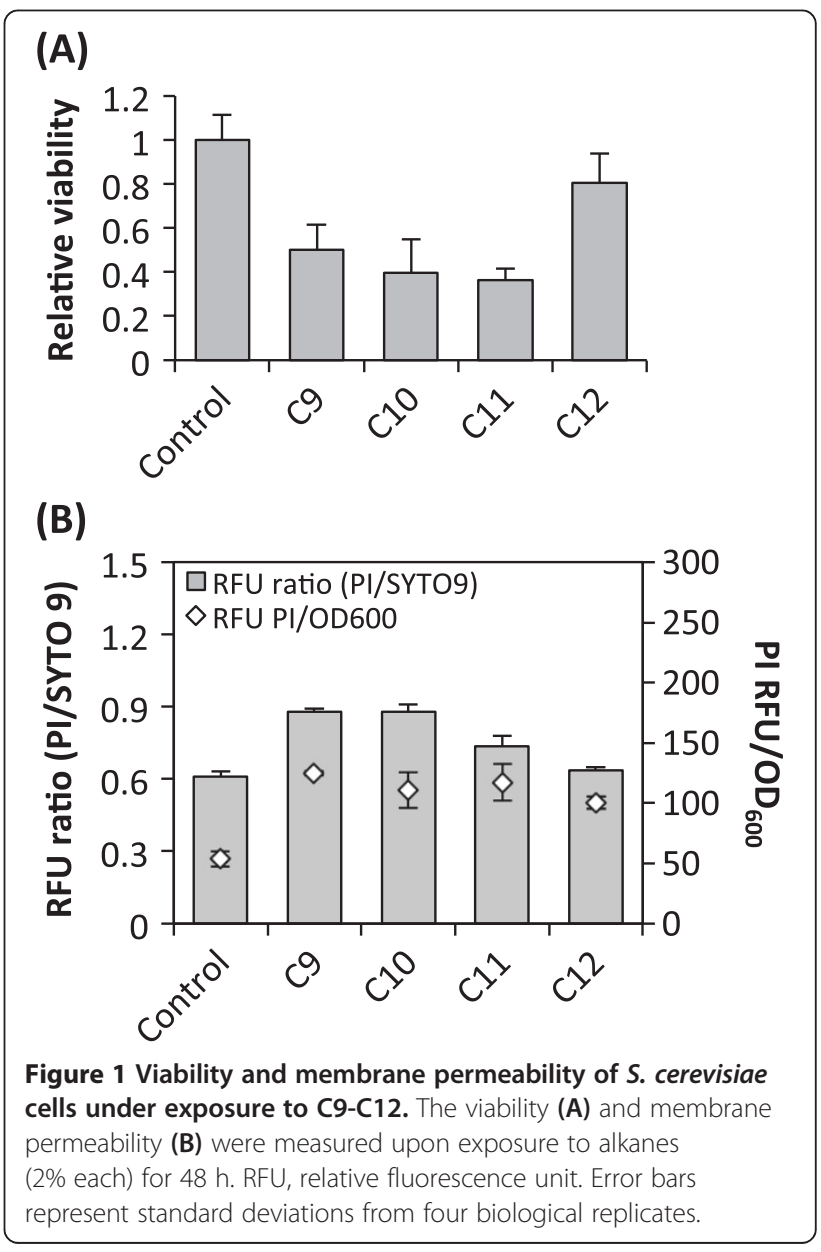


damage was not apparent on cell surface upon exposure to $2 \%$ C9, which is in agreement with de Smet and coworkers' study [20]. Based on this result, we hypothesized that alkane toxicity might be attributed at least partly to intracellular accumulation. GC analyses show that a peak corresponding to each alkane was detected from alkanes-treated cells, while control cells without alkanes exhibited a negative signal (Additional file 2: Figure S2). To measure intracellular alkane amounts, we normalized GC peak areas to the internal standard and the total protein amount. Among C9 to C12, C10 showed the highest intracellular level, followed by $\mathrm{C} 9$ and C11, and C12 level was the lowest which was $90 \%$ lower than C10 level (Figure 2). These results indicate that alkanes were accumulated in cells, which likely contributed to alkane toxicity. According to Inoue and Kawamoto [21,22], toxicity of a compound is related to coefficient of octanol-water partition $\left(\log P_{\text {ow }}\right)$. Gill and coworkers reported that higher aliphatic chain alkanes tend to show lower toxicity [23]. In our study, C12 (log $P_{\text {ow }}$ 7.0) showed lower toxicity than C9 to C11 $\left(\log P_{\text {ow }}\right.$ $<6.0$ ), which could be partially due to low accumulation of C12 in S. cerevisiae.

\section{Genome-wide gene expression profiles upon exposure to biofuel alkanes}

To understand cellular mechanisms underlying $S$. cerevisiae response to C9-12, transcriptome profiles were generated using whole-genome $S$. cerevisiae microarrays. Significantly regulated genes (fold changes $\geq 2.0$, and

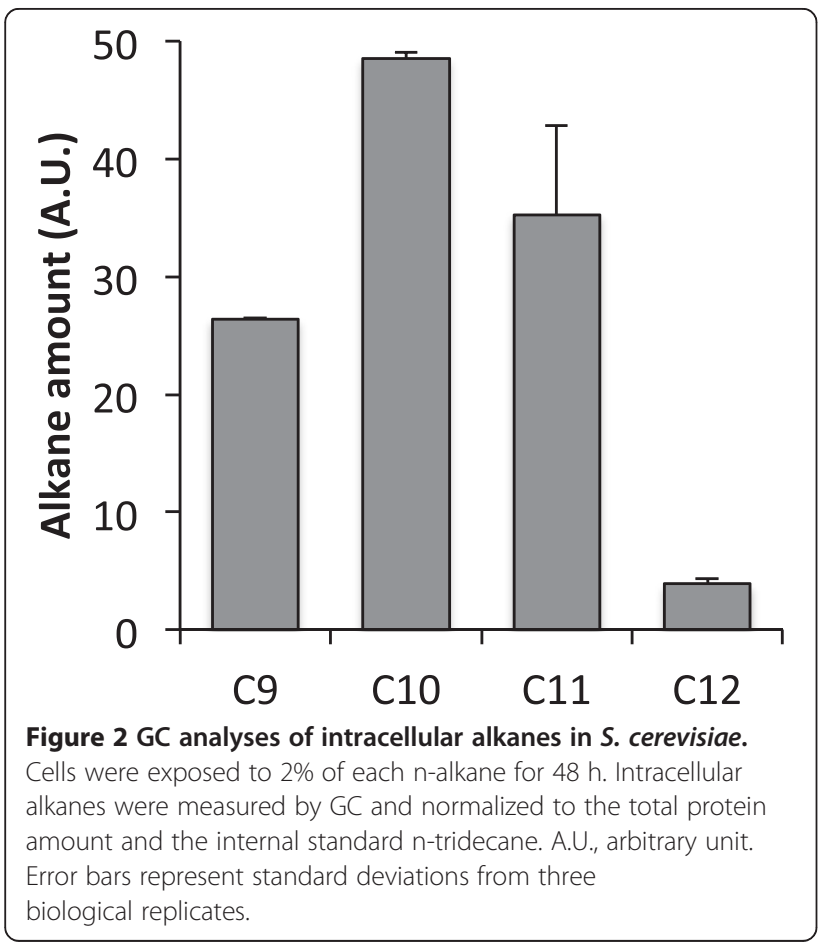

p values $<0.05$ ) are summarized in Additional file 3 . The data discussed in this publication have been deposited in NCBI's Gene Expression Omnibus and are accessible through GEO Series accession number GSE38653. In addition, quantitative PCR (qPCR) was carried out to validate the microarray data (Additional file 3).

Our transcriptome analysis indicated that 526 (C10 for $24 \mathrm{~h}$ ), 692 (C9 for $48 \mathrm{~h}$ ), 428 (C10 for $48 \mathrm{~h}$ ), and 119 (C11 for $48 \mathrm{~h}$ ) genes were differentially regulated, while $5(24 \mathrm{~h})$ and $8(48 \mathrm{~h})$ genes were regulated by $\mathrm{C} 12$ (Additional file 3). Functional analysis of those genes suggested that $\mathrm{C} 11$ primarily regulated genes related to heat shock response and sugar transport, whereas C9 and $\mathrm{C} 10$ induced a range of similar groups of genes. Therefore, to look into cellular mechanisms commonly stimulated by alkanes, we analyzed the genes regulated by both $\mathrm{C} 9$ and C10. 147 genes were significantly regulated by both C9 and C10. Among the 147 genes, 105 genes were induced by both $\mathrm{C} 9$ and $\mathrm{C} 10$, whereas 42 genes were repressed by both $\mathrm{C} 9$ and C10 (except for COS12 gene which was down-regulated by $\mathrm{C} 10$ but upregulated by $\mathrm{C} 9$ ). To further understand gene functions, we functionally classified the aforementioned regulated genes using MIPS tools (http://funspec.med.utoronto.ca/) [24]. Primary functional classes of the regulated genes were (i) efflux pumps, (ii) stress response, (iii) fatty acid, lipid, and derivatives biosynthesis, and (iv) hexose transport, ergosterol biosynthesis, and thiamine biosynthesis (Table 1). The genes in these classes were mostly induced by $\mathrm{C} 9$ and $\mathrm{C} 10$.

First of all, notably, four plasma membrane efflux pump genes YOR1, SNQ2, PDR5, and PDR15 were significantly induced by alkane stress. Plasma membrane efflux pumps recognize and extrude a large spectrum of functionally and structurally unrelated drugs such as oligomycin, mycotoxins, and anticancer drugs [25-28]. That is, when cytotoxic compounds cross the plasma membrane to enter cells, a number of efflux pumps help to protect cells from unwanted or damaging xenobiotics. According to Ernst and coworkers [29], efflux pumps serve as a defense line mediating pleiotropic drug resistance (PDR). Therefore, the result that efflux pump genes were significantly induced here suggested that they might also provide protection to the cells against alkanes.

Other than efflux pump genes, genes involved in stress response for radical detoxification, fatty acid and lipid synthesis, and hexose transport were also induced by alkanes. For example, GRE genes associated with multiple stresses [30] and heat shock protein gene HSP12 were induced up to 16-fold, and fatty acid synthase genes FAS1 and FAS2 were induced over 4-fold. Acetyl-CoA carboxylase gene $A C C 1$ was up-regulated about 6-fold. Acc1p is a biotin-containing enzyme that participates 
Table 1 MIPS functional classification of genes differentially regulated by both $\mathrm{C} 9$ and $\mathrm{C} 10$

\begin{tabular}{|c|c|c|}
\hline Functional category & k & $f$ \\
\hline \multicolumn{3}{|l|}{ Up-regulated } \\
\hline fatty acid metabolism & 5 & 24 \\
\hline phospholipid metabolism & 7 & 68 \\
\hline oxidative stress response & 6 & 55 \\
\hline tetracyclic and pentacyclic triterpenes & 5 & 36 \\
\hline cellular import & 7 & 90 \\
\hline lipid/fatty acid transport & 5 & 44 \\
\hline ABC transporters & 4 & 28 \\
\hline lipid, fatty acid and isoprenoid metabolism & 8 & 133 \\
\hline degradation of glutamate & 2 & 4 \\
\hline metabolism of amino acid-derived secondary products & 2 & 4 \\
\hline NAD/NADP binding & 4 & 36 \\
\hline C-compound and carbohydrate metabolism & 10 & 223 \\
\hline heat shock response & 3 & 20 \\
\hline chemical agent resistance & 3 & 22 \\
\hline S-adenosyl-methionine-homocysteine cycle & 2 & 7 \\
\hline pH stress response & 2 & 8 \\
\hline oxidation of fatty acids & 2 & 9 \\
\hline transport ATPases & 4 & 53 \\
\hline \multicolumn{3}{|l|}{ Down-regulated } \\
\hline biosynthesis of thiamine & 6 & 110 \\
\hline transport facilities & 5 & 87 \\
\hline degradation of lysine & 2 & 5 \\
\hline degradation of glycine & 2 & 6 \\
\hline transport routes & 2 & 11 \\
\hline metabolism of thiamine & 3 & 43 \\
\hline tetrahydrofolate-dependent C-1-transfer & 2 & 13 \\
\hline
\end{tabular}

Note: functional classification was generated by the online program FunSpec (http://funspec.med.utoronto.ca/) with p-value cut-off of 0.01. k, number of genes from the input (105 up-regulated genes, and 42 down-regulated genes) in the given category, $\mathrm{f}$, number of genes total in the given category.

in fatty acid synthesis by converting acetyl-CoA to malonyl-CoA, which is a rate-limiting step in the fatty acid synthesis pathway [31]. In addition, INO1 gene was induced over 50 -fold, which is associated with synthesis of phosphatidylinositol by using glucose-6-P and CDPDAG as precursors [32].

Upon alkanes exposure, six transcription factor genes, ASH1, GAL4, NRG2, MGA2, YPRO15C, and THI2, were differentially regulated. Because changes in gene expression levels of transcription factors frequently do not correlate with changes in their regulatory activities, to further identify transcription factors with perturbed regulatory activities under alkane exposure, we analyzed our transcriptome data using Network Component Analysis (NCA). NCA is an algorithm that calculates relative activities of transcription factors from gene expression data [13]. NCA results suggested that regulatory activities of nine transcription factors were changed upon exposure to C9 (48 h) and C10 (24 h, and $48 \mathrm{~h}$ ) (Table 2). Among the nine transcription factor genes identified from NCA, the activities of six transcription factors were increased, and the activities of three were decreased. To further understand regulatory relationships between transcription factors and the genes involved in $\mathrm{C} 9$ and $\mathrm{C} 10$ response, a regulation matrix was generated by YEASTRACT (http://www.yeastract.com/) [33]. In the generated transcription networks (Figure 3), INO2 showed regulation of genes associated with efflux pumps, stress response, lipid metabolism and ergosterol biosynthesis. Hereby, we predicted that INO2 would most likely be one of the key transcription factors that regulate gene networks in response to alkanes exposure in $S$. cerevisiae. Further, the three repressed transcription factors (i.e. CIN5, FHL1, and HAP1) likely up-regulate their target genes such as GRE2, GND2, and ERG6 (Figure 3). Therefore, there is a possibility that the aforementioned transcription factors induce genes associated with efflux pumps, stress response, and lipid metabolism in S. cerevisiae upon exposure to both C9 and C10.

Based on our transcriptome data, and the functions and physiological roles of the regulated genes, we hypothesized that $S$. cerevisiae responded to alkanes stresses through integrative mechanisms (depicted in Figure 4). First, efflux pump genes (YOR1, SNQ2, PDR5, and PDR15) are induced to export intracellular alkanes to reduce alkane toxicity. Second, alkanes exposure generates radicals and induces stress-responsive genes towards radical detoxification. Alkane molecules penetrate cell wall, disperse into cytoplasmic membrane, and generate radicals (e.g. reactive oxygen species (ROS), and $\mathrm{NO}$ ), while stress-responsive genes (GRE1, GRE2, CTT1, $H S P 12, T S A 2$, and $Y H B 1)$ are induced to detoxify radicals including ROS. Notably, in line with this hypothesis,

Table 2 Transcription factors with activity changes, predicted by Network Component Analysis (NCA)

\begin{tabular}{llccc}
\hline Transcription factors & \multicolumn{3}{c}{ Regulatory activity } \\
\cline { 2 - 5 } & & $\mathbf{C 1 0 - 2 4} \mathbf{~}$ & $\mathbf{C 1 0 - 4 8 ~}$ & $\mathbf{~} \mathbf{~ 9 - 4 8 ~} \mathbf{~}$ \\
\hline YKL112W & ABF1 & +3 & +3 & +3 \\
\hline YKR099W & BAS1 & +3 & +3 & +4 \\
\hline YDR123C & INO2 & +12 & +18 & +19 \\
\hline YOL108C & INO4 & +3 & +4 & +3 \\
\hline YGL162W & SUT1 & +4 & +5 & +5 \\
\hline YDR207C & UME6 & +6 & +4 & +4 \\
\hline YOR028C & CIN5 & -2 & -3 & -10 \\
\hline YPR104C & FHL1 & -3 & -4 & -8 \\
\hline YLR256W & HAP1 & -3 & -3 & -7
\end{tabular}

Note: C10-24 h, cells exposed to $\mathrm{C} 10$ for $24 \mathrm{~h}$ compared to cells without alkane exposure for $24 \mathrm{~h}, \mathrm{C}$ - or C10-48 h, cells exposed to C9 or C10 for $48 \mathrm{~h}$ compared to cells without alkane exposure for 48 h. +, upregulation, - , downregulation. 


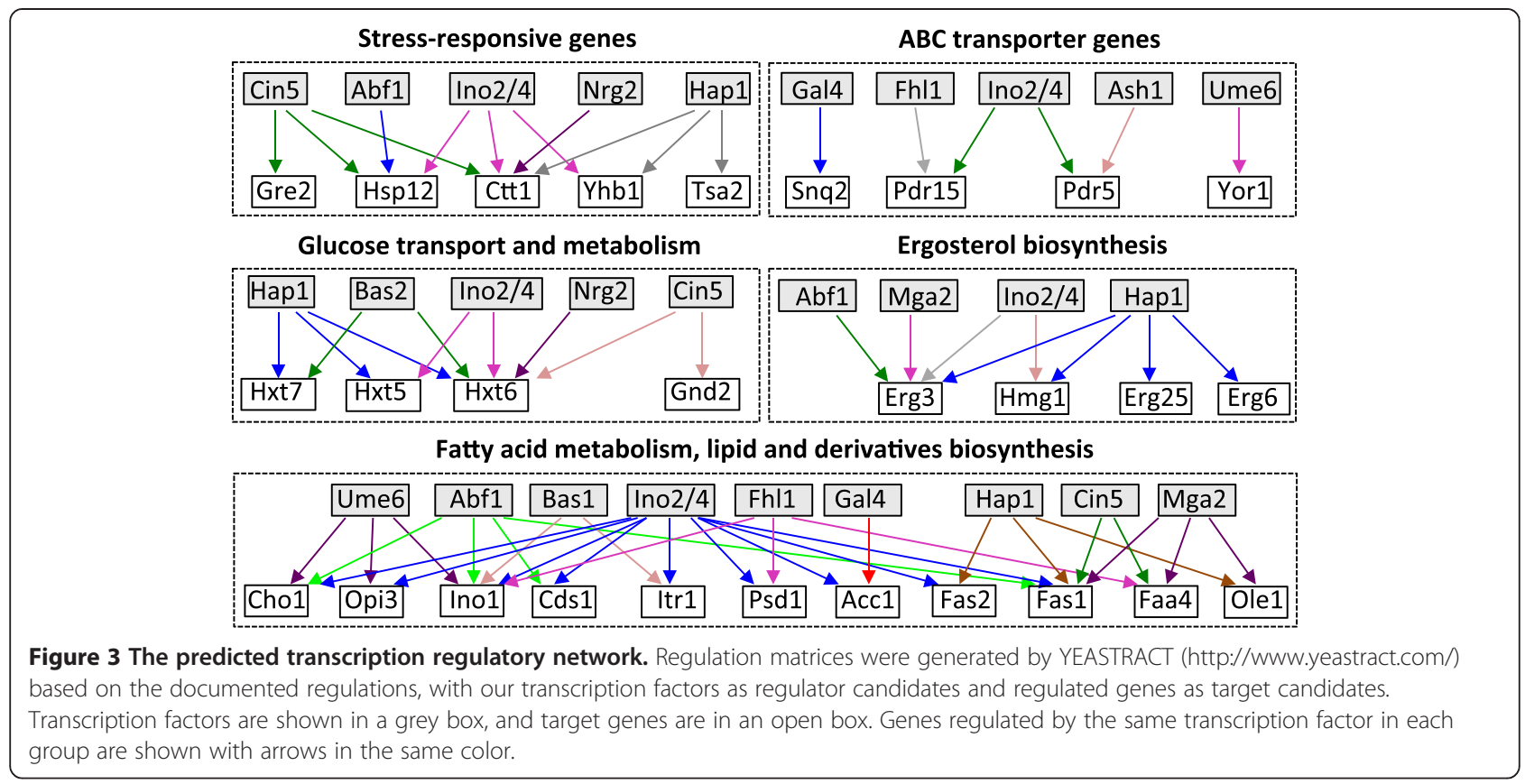

we observed that ROS levels were increased by $110 \%$ and $78 \%$ in S. cerevisiae upon exposure to $\mathrm{C} 9$ and $\mathrm{C} 10$, respectively (Additional file 3). Such increase in ROS levels is most likely correlated with alkanes toxicity. Third, more energy sources are generated through acceleration of glucose and fatty acid metabolism. Hexose transport genes (HXT5, HXT6, and HXT7) are induced to increase glucose import. Thereafter, energy sources (FADH, NADH, NADPH, and ATP) are enriched through enhanced glucose metabolism involving pentose phosphate pathway (GND2), $\beta$-oxidation of fatty acids (FAA4, and POT1), and ALD3-involved pathways. ATP may be used for alkanes export by efflux pumps. Fourth, the increase in glucose import and consumption aids in modifying membrane components (i.e. lipid, and ergosterol), thereby helping cells to adapt to alkanes exposure. Enriched glucose inside the cells may lead to the synthesis of more fatty acids, lipids, and derivatives (i.e. phospholipid, glycerophospholipid, phosphatidylinositol, and phosphatidylcholine), contributed to by such induced

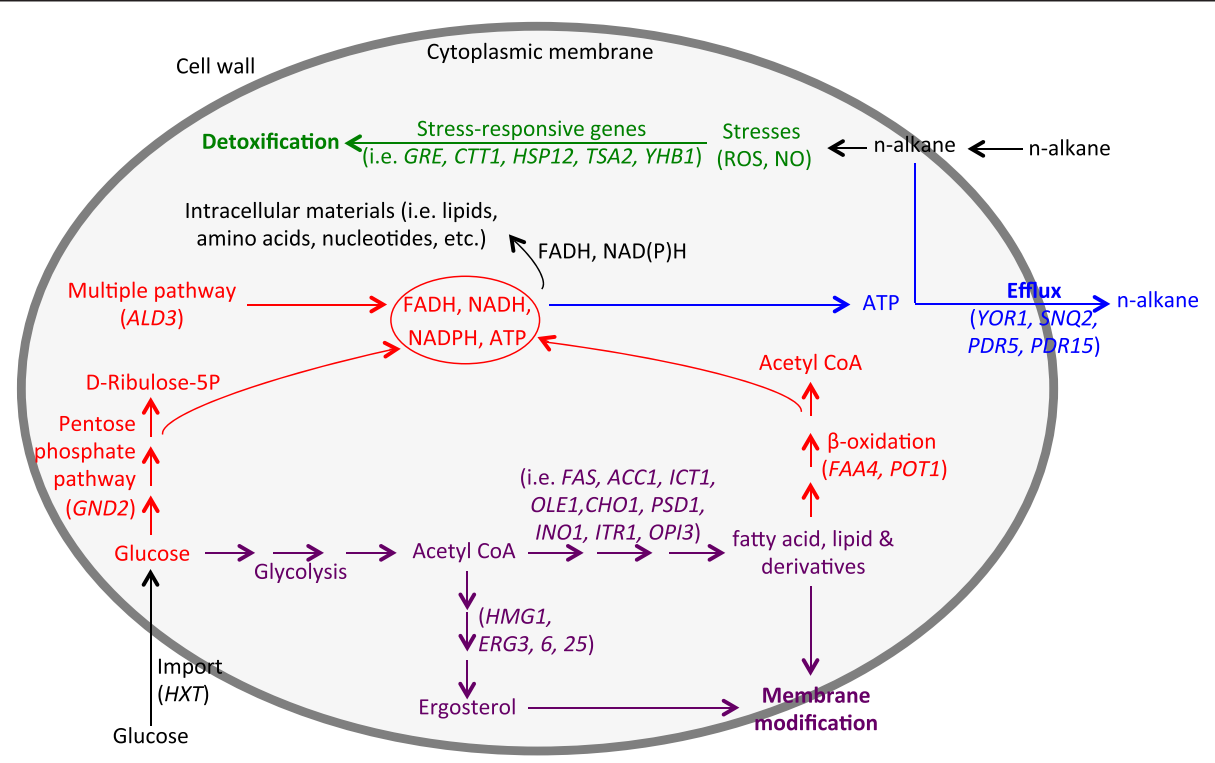

Figure 4 Schematic representation of hypothesized mechanisms of cellular response to alkanes in S. cerevisiae. The mechanisms include alkane efflux (blue), detoxification of radicals (green), energy supply (red), and membrane modification (purple). 
genes as FAS1, FAS2, ACC1, ICT1, OLE1, CHO1, PSD1, INO1, ITR1, and OPI3. Further, ergosterol biosynthesis is enhanced by the four induced genes HMG1, EGR3, ERG6, and ERG25. Such a change in the expression of genes involved in lipid and ergosteols metabolism may lead to membrane modification, possibly facilitating adaptation to alkanes exposure.

\section{Overexpression of efflux pumps leads to improved tolerance to biofuel alkanes}

Among the aforementioned mechanisms, we were interested in further identifying roles of plasma membrane efflux pumps in alkane tolerance and export because (i) plasma membrane efflux pumps are the first line of defense against drugs or organics, and (ii) microbial efflux pumps reportedly serve as a direct mechanism to improve biofuel tolerance [4,34-37] as well as productivity [4]. In fungi, efflux pumps, especially ATP-binding cassette $(\mathrm{ABC})$ efflux pumps (e.g. Pdr5p and Snq2p in $S$. cerevisiae, Cdr1p and Cdr2p in Candida albicans), which contribute to multidrug efflux resistance, have been most extensively studied [25-28,38]. In our study, all the four efflux pumps induced by alkanes are $A B C$ transporters, and considered as major pleiotropic drug transporters that comprise a PDR network in yeast [39]. Among them, Snq2p, Pdr5p, and Pdr15p belong to a pleiotropic drug resistance (PDR) protein subfamily, and Yorlp belongs to a multidrug resistance-associated protein (MRP) subfamily. Their protein architecture contains multiple transmembrane domains (TMDs) that bind chemical compound and provide a translocation channel, and nucleotide-binding domains (NBDs) that couple ATP hydrolysis to substrate transport. Given that the induced $A B C$ efflux pumps play roles in reducing intracellular alkane accumulation, tolerance of S. cerevisiae cells against alkanes could be improved. Hence, we formed a hypothesis that the significantly induced efflux pumps might also help cells to reduce the intracellular alkane accumulation and thus, provide protection to cells against alkanes.

To identify roles of each of the induced efflux pumps in reducing intracellular alkane levels and improving alkane tolerance levels, we constructed triple knock-out mutants of efflux pumps, BYL251K (yor1 1 snq $2 \Delta$ pdr $5 \Delta$ ) and BYL2515K (snq2 $\Delta p d r 5 \Delta p d r 15 \Delta)$. Subsequently, we cloned the four efflux pump genes (YOR1, SNQ2, PDR5, and PDR15) into plasmid pYES2 respectively, and transformed recombinant plasmids into the triple mutants. Under control of the galactose-inducible promoter $\mathrm{P}_{\mathrm{GAL1}}$ and exposure to alkanes, the aforementioned efflux pump genes (YOR1, SNQ2, and PDR5) were expressed in the triple mutant BYL251K respectively, and PDR15 was expressed in the triple mutant BYL2515K. Cell tolerance and intracellular alkane levels were then analyzed (Figure 5, and Additional file 4: Figure S3). Figure 5A shows that there was similar growth among the BYL251K cells with pYES2 (control) and with SNQ2 or PDR5 in an alkane-free induction medium. Upon exposure to C10, cell growth of the triple mutant BYL251K expressing each of Snq2p and Pdr5p was significantly enhanced, compared to BYL251K with the plasmid pYES2 (Figure 5B). This result suggested that expression of Snq2p and Pdr5p, respectively, increased cellular tolerance to C10. Furthermore, Figure 5C shows that upon $24 \mathrm{~h}$ exposure to $\mathrm{C} 10$, intracellular $\mathrm{C} 10$ amount was lowered by $25 \%$ and $33 \%$ in the triple mutant BYL251K expressing either Snq2p or Pdr5p. This result indicated a crucial role of Pdr5p and Snq2p in reducing the intracellular $\mathrm{C} 10$ levels, consistent with the hypothesis on the role of efflux pumps in improving alkane tolerance as mentioned above. Furthermore, we tested growth of cells expressing Pdr5p and Snq2, respectively, under $5 \% \mathrm{C} 11$. In support of our hypothesis, the triple knock-out mutant BYL251K expressing the efflux pump Snq2p or Pdr5p showed much higher cell density than the control cells, even though C11 did not induce expression of those efflux pumps according to our microarray data. This result suggested that tolerance to $\mathrm{C} 11$ was significantly improved by the efflux pumps Snq2p and Pdr5p. Further, Figure 5C shows that intracellular C11 levels were lowered by $87.4 \%$ and $94.4 \%$ in the triple mutant BYL251K expressing Snq2p and Pdr5p, respectively. This result indicated a crucial role of Pdr5p and Snq2p in reducing the intracellular C11 levels, consistent with the aforementioned hypothesis on the role of efflux pumps in improving the tolerance to C11. Note that the Resistance-Nodulation-Division (RND) efflux pumps such as AcrAB-TolC pump, Mex pumps, Ttg pumps from Gram-negative bacteria have been previously studied as an effort to improve tolerance to biofuels in bacteria [34,35,37,40]. However, the RND pumps utilize proton or sodium gradients as a source of energy $[41,42]$ instead of ATP by ABC efflux pumps. This suggests that yeast and gram-negative bacteria employ different types of efflux pumps for improving the biofuel tolerance.

However, even though the efflux pump genes (YOR1, SNQ2, PDR5, and PDR15) were all induced by both C9 and $\mathrm{C} 10$ according to our microarray data (Additional file 3), there was no improvement in tolerance against C9 by complementation of each of the four efflux pumps. In addition, there was no improvement in tolerance against $\mathrm{C} 10$ and $\mathrm{C} 11$ by complementation of Yor1p or Pdr15p. This outcome was probably due to variance in substrate binding efficacy of various efflux pumps, and/or the involvement of such mechanisms as stress response and membrane modification, in addition to efflux pumps, in cellular tolerance to C9. Engineering 

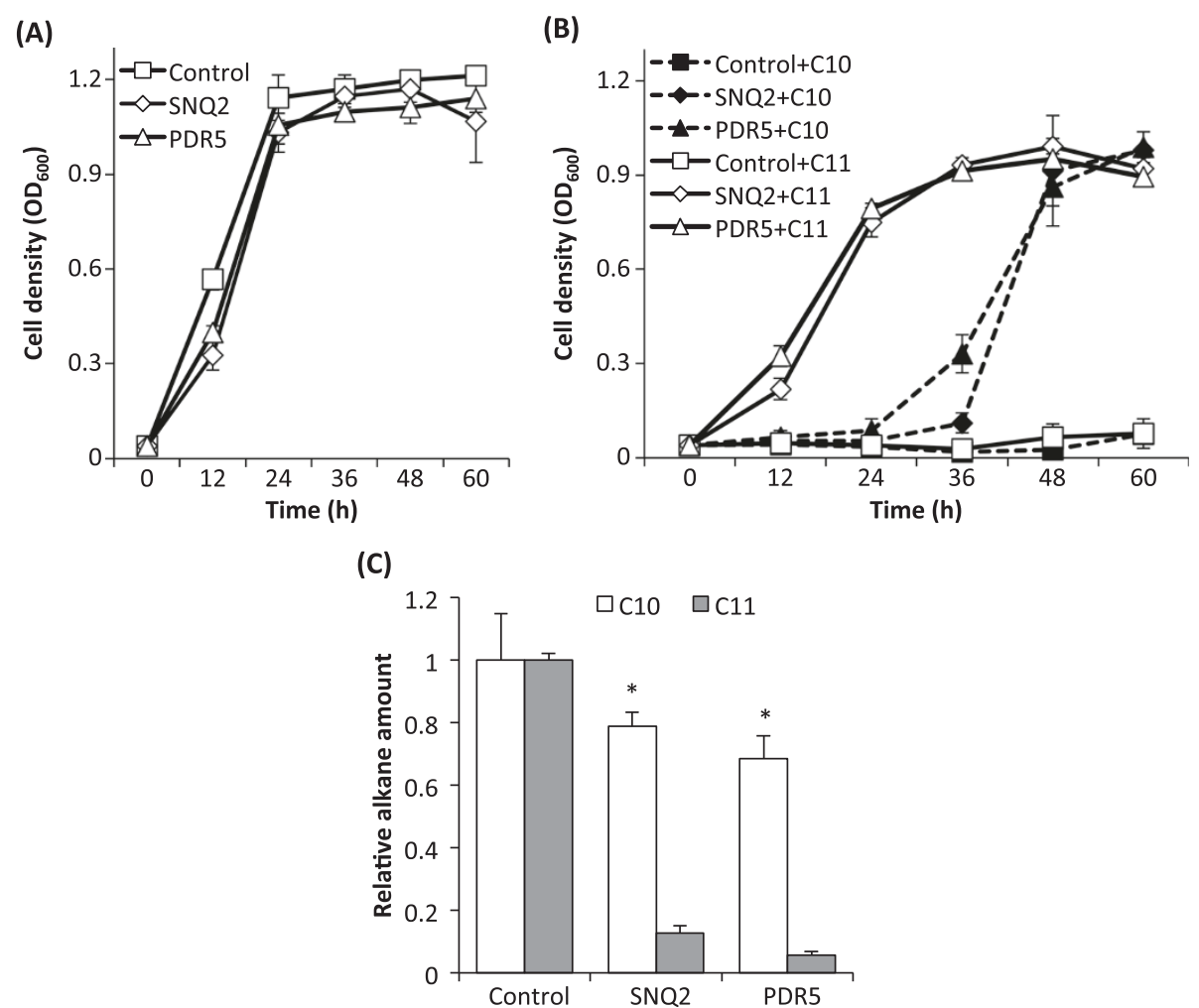

Figure 5 Growth of triple knock-out mutants expressing efflux pumps and their intracellular alkane levels under alkane exposure. The triple mutant BYL251K (yor $1 \Delta$ snq2 $\Delta$ pdr5 4 ) expressing Snq2p and Pdr5p, respectively, were grown in induction media without alkanes (A) or with $2 \% \mathrm{C} 10$ or $5 \% \mathrm{C} 11$ (B). OD 600 values represent cell density. After $24 \mathrm{~h}$ exposure, intracellular C10 and C11 levels were measured by GC and normalized to the total protein amount and the internal standard C12 (C). Alkane amount in control cells was set as 1. Control, the triple mutant BYL251K with pYES2. Error bars represent standard derivations of at least three biological replicates. ${ }^{*}$, statistically significant difference (Student $t$ test, $\mathrm{p}<0.05)$ compared to cells with pYES2.

approaches such as site-mutation, evolutionary engineering, and gene shuffling can be employed to improve substrate binding efficacy [10,43-45]. Other than the indigenous efflux pumps in S. cerevisiae, efflux pumps from other microbes can also be introduced to improve tolerance against biofuel alkanes. In addition, future studies could include use of other cellular mechanisms besides efflux pumps as an effort to improve alkane tolerance.

\section{Conclusions}

To our knowledge, this is the first report providing the evidence of the direct linkage between alkane tolerance and indigenous efflux pumps, and of being able to develop alkane-tolerant $S$. cerevisiae by directly harnessing indigenous efflux pumps. Herein, we first confirmed that C9, C10, and C11 were significantly toxic and accumulated in S. cerevisiae. Transcriptome analyses suggested that C9 and C10 induced a range of cellular mechanisms such as efflux pumps, membrane modification, radical detoxification, and energy supply. Since efflux pumps could possibly aid in alkane export, thereby reducing the cytotoxicity, we formed the hypothesis that those induced efflux pumps could contribute to alkane tolerance. In support of this hypothesis, we demonstrated the roles of the efflux pumps Snq2p and Pdr5p in reducing the intracellular levels of $\mathrm{C} 10$ and $\mathrm{C} 11$, and enhancing the tolerance levels against $\mathrm{C} 10$ and $\mathrm{C} 11$. This result provided the evidence that Snq2p and Pdr5p were associated with alkane export and tolerance in S. cerevisiae. We believe that the results here provide valuable insights into designing microbial engineering strategies to improve cellular tolerance for highly efficient alkane biofuel production.

\section{Methods}

\section{Strains, chemicals and plasmids}

S. cerevisiae BY4741 (MATa; his3 $\Delta 1$; leu2 $\Delta 0$; met15 $\Delta 0$; ura $3 \Delta 0$ ) was obtained from ATCC (American Type Culture Collection), and gene deletion mutants were constructed in this study (Additional file 3). Yeast extract and peptone were obtained from BD (NJ, USA). Alkanes and other chemicals were obtained from SigmaAldrich unless specifically mentioned. pUG6 and pSH47 
were used for gene deletion and marker rescue in yeast cells. Plasmid pYES2 (Invitrogen, Grand Island, NY, USA) was used for gene expression in yeast cells. E. coli Top10 was used for gene cloning.

\section{Growth conditions}

S. cerevisiae strains were grown in YPD medium (Yeast extract $10 \mathrm{~g} / \mathrm{l}$, Peptone $20 \mathrm{~g} / \mathrm{l}$, and D-Glucose $20 \mathrm{~g} / \mathrm{l}$ ), minimum medium (Yeast Nitrogen Base $6.7 \mathrm{~g} / \mathrm{l}$, DGlucose 20 g/l, L-Leucine 0.2 g/l, Uracil 0.1 g/l, 2\% nalkanes; Yeast Nitrogen Base 6.7 g/l, D-Glucose 20 g/l, Yeast Synthetic Drop-out Medium Supplements-Ura ${ }^{-}$ $1.92 \mathrm{~g} / \mathrm{l}$ ), or induction medium (Yeast Nitrogen Base $6.7 \mathrm{~g} / \mathrm{l}$, Raffinose $10 \mathrm{~g} / \mathrm{l}$, Galactose $20 \mathrm{~g} / \mathrm{l}$, and Yeast Synthetic Drop-out Medium Supplements-Ura $1.92 \mathrm{~g} / \mathrm{l}$ ) at appropriate temperatures. $E$. coli cells were grown in LB broth at $37^{\circ} \mathrm{C}$. Geneticin G418 (PAA Laboratories $\mathrm{GmbH}, \mathrm{AT}$ ) or Ampicillin was used at $200 \mu \mathrm{g} / \mathrm{ml}$ and $100 \mu \mathrm{g} / \mathrm{ml}$, respectively.

\section{Cell viability, membrane integrity and reactive oxygen species (ROS)}

S. cerevisiae BY4741 cells were grown overnight in YPD, diluted into minimal medium with amino acids which contains each of $2 \%$ alkanes (C9-C12) with an initial $\mathrm{OD}_{600}$ of 0.2 , and followed by shaking incubation at $28^{\circ} \mathrm{C}$, and viable cells measurement by CFU (Colony Forming Unit) counting. To investigate whether these alkanes resulted in membrane damage, membrane permeability was analyzed by cell staining. After exposure to alkanes, cells were stained with fluorescent nucleic acid stains SYTO 9 and propidium iodide (PI) (Invitrogen) that only penetrates cells with damaged membrane. Finally, signal intensity in cells was measured using Synergy HT multi-mode microplate reader (Biotek, VT, USA) at wavelength of $485 / 20 \mathrm{~nm}$ (excitation), 528/ $20 \mathrm{~nm}$ (SYTO 9 emission), and 645/40 nm (PI emission). To investigate reactive oxygen species (ROS) generation, alkane-treated cells were collected and stained by CellROX $^{\circledR}$ Green Reagent (Life Technologies). Fluorescence signals were measured by TECAN Infinite 200 microplate reader at wavelength of $485 \mathrm{~nm}$ (excitation)/ $535 \mathrm{~nm}$ (emission) and normalized to cell density $\left(\mathrm{OD}_{600}\right)$.

\section{Alkane extraction and detection}

Alkanes were extracted by chloroform-methanol extraction method [46] with modifications. Firstly, yeast cells were collected and washed with $50 \mathrm{mM}$ Tris.Cl (pH 7.4) then resuspended in $0.4 \mathrm{ml}$ chloroform/methanol mixture (v/v: 2/1). Subsequently, $0.3 \mathrm{~g}$ acid-washed glass beads (425-600 $\mu \mathrm{m}$, Sigma-Aldrich) were added into each sample and cells were lysed using FastPrep-24 (MP Biomedicals, Solon, OH, USA). Then, after the lysate was centrifuged at $4^{\circ} \mathrm{C}$ at maximum speed for $10 \mathrm{~min}$, an appropriate volume of chloroform and $50 \mathrm{mM}$ Tris. $\mathrm{Cl}$ were added to the supernatant for extraction. Finally, the chloroform phase with alkanes was transferred into glass vials for Gas Chromatography (GC) analysis. This was performed using Agilent GC 7890A system (Agilent Technologies, Santa Clara, CA, USA) under the following conditions. With Agilent HP-5 GC column, oven temperature was started at $40^{\circ} \mathrm{C}$ for $30 \mathrm{sec}$, and was ramped from $40^{\circ} \mathrm{C}$ to $220^{\circ} \mathrm{C}$ at $40^{\circ} \mathrm{C} / \mathrm{min}$, and FID detector temperature remained at $275^{\circ} \mathrm{C}$. A mixture of $\mathrm{n}$ alkanes (10 ppm each) was used as the standard for GC analysis.

\section{RNA preparation, transcriptome analysis, and quantitative PCR}

Total RNA was extracted with lyticase for cell wall disruption using RNeasy Mini Kit (Qiagen, Hilden, Germany). Total RNA concentration and integrity were measured using NanoDrop (Thermo Scientific, Wilmington, DE, USA) and Agilent Bioanalyzer 2100, respectively. Subsequently, $200 \mathrm{ng}$ of the qualified total RNA was transcribed and labeled using Agilent RNA Spike-in Kit and Low Input Quick Amp Labeling Kit (two colors). The Cy3- or Cy5-labeled cRNA was hybridized onto Yeast (V2) Gene Expression Microarray $(8 \times 15 \mathrm{~K})$ at $65^{\circ} \mathrm{C}$ for $17 \mathrm{~h}$, using Agilent GE Hybridization Kit. Thereafter, the microarray slide was scanned using Agilent Microarray Scanner G2505B. The two-color scanning data were extracted using Agilent Feature Extraction software (v10.5) and analyzed using GeneSpring GX 11. From two biological replicates, differentially expressed genes were selected based on their $\mathrm{p}$ values $(<0.05, t$-test $)$ and fold changes $(\mathrm{FC})$ in $\mathrm{n}$-alkanetreated cells in comparison to control cells $(\geq 2.0)$.

To validate the microarray data, quantitative PCR (qPCR) was performed with primers targeting the selected genes (Additional file 3) and SsoFast ${ }^{\mathrm{tm}}$ EvaGreen $^{\circledR}$ Supermix using iQ5 real-time PCR detection system (Bio-Rad). The qPCR data analysis was conducted with $A C T 1$ as a reference gene using Bio-Rad iQ5 optical system software.

\section{Network component analysis (NCA)}

The Network Component Analysis is an algorithm that calculates relative activities of transcription factors from gene expression data [13]. In brief, a matrix of gene expression data $([E])$, which contain genes in rows and experimental conditions in columns, are prepared for NCA analysis. Subsequently, the $[E]$ matrix is decomposed into two matrices, a connectivity strength (CS) matrix ([A]) with transcription factors (columns) and genes (rows) and a transcription factor activity (TFA) matrix ([P]) with transcription factors (rows) an experimental conditions (columns). In our study, to perform NCA 
analysis, first, genes in the gene expression data matrix ([E]) were built from 147 genes (fold changes $\geq 2.0$, and $\mathrm{p}$ values $<0.05$ ), up- or down-regulated under the three conditions ( $\mathrm{C} 10$ vs. control at $24 \mathrm{~h}$ and $48 \mathrm{~h}$, and $\mathrm{C} 9$ vs. control at $48 \mathrm{~h}$ ); second, the connectivity strength (CS) matrix [A] from Lee and coworkers [47] was used to primarily define connectivity relations between the genes and the transcription factors; third, the final TFA matrix $[\mathrm{P}]$ was deduced by NCA.

\section{Yeast gene deletion}

Gene deletion was carried out as previously described [48]. Briefly, loxP-Kan-loxP gene disruption cassettes were amplified using PCR with primers as shown in Additional file 3 and pUG6 as the template, which were then purified using QIAquick Gel Extraction Kit (Qiagen). The purified cassettes were used for yeast transformation as previously described. The colonies were screened on YPD plates containing $200 \mu \mathrm{g} / \mathrm{ml} \mathrm{G418}$ and confirmed by PCR. To perform marker rescue, pSH47 was transformed into mutants, selected on minimal medium ( $\left.\mathrm{Ura}^{-}\right)$plates, and followed by Cre/loxP-mediated marker removal in induction medium. Finally, selection for the loss of marker was performed in YPD containing $1 \mathrm{mg} / \mathrm{ml} \mathrm{5-fluoro-orotic} \mathrm{acid} \mathrm{(Thermo}$ Scientific).

\section{Plasmid construction and analyses of yeast strains overexpressing efflux pump genes}

The gene specific primers (Additional file 3) and iProof High-Fidelity DNA Polymerase (Bio-Rad) were employed to amplify target genes by PCR with S. cerevisiae BY4741 genomic DNA as a template. DNA fragments were purified and cloned into an expression vector pYES2. The recombinant plasmids were prepared using QIAprep Miniprep Kit (Qiagen), transformed into S. cerevisiae mutants by electroporation [49], and followed by selection on Geneticin-containing minimal medium plates. To investigate effects of mutants expressing efflux pumps on cell growth and alkane accumulation, cells harboring a recombinant plasmid were grown in an induction medium plus alkanes. Subsequently, optical density of cells at $600 \mathrm{~nm}$ $\left(\mathrm{OD}_{600}\right)$ and intracellular alkanes were measured.

\section{Additional files}

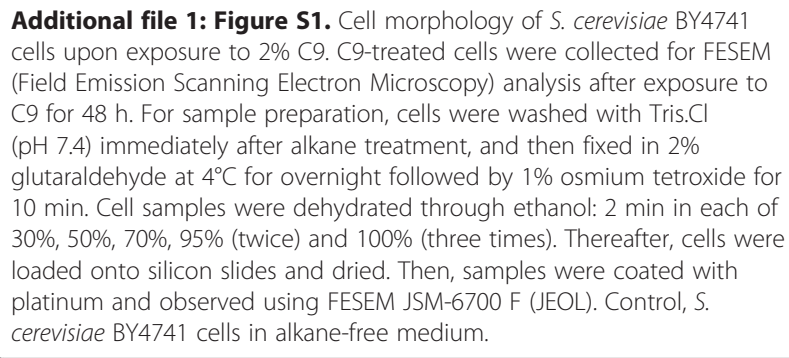

Additional file 2: Figure S2. GC chromatograms of alkanes extracted from S. cerevisiae BY4741 cells. Control, cells without alkanes, IS, internal standard, C13, n-tridecane. Peaks were indicated by arrows.

Additional file 3: Table S1. List of genes differentially regulated in S. cerevisiae upon exposure to alkanes. This table contains 7 sub-tables (A-G): A. Genes differently regulated upon exposure to C10 (24 h, and 48 h) and C9 (48 h); B. C9-48 h; C. C10-24 h; D. C10-48 h; E. C11-48 h; F. C12-24 h. G. C12-48 h. Table S2. Validation of microarray data by qPCR upon exposure to C10 for 48 h. Table S3. ROS levels in S. cerevisiae upon exposure to C9 and C10 for $24 \mathrm{~h}$. Table S4. Yeast strains and plasmids used in this study. Table S5. Primers used in this study.

Additional file 4: Figure S3. GC chromatograms of $\mathrm{C} 10$ and $\mathrm{C} 11$ from S. cerevisiae BYL251K cells. IS, internal standard. Peaks were indicated by arrows.

\section{Abbreviations}

NCA: Network component analysis; C9: n-nonane; C10: n-decane; C11: n-undecane; C12: n-dodecane.

\section{Competing interests}

The authors declare that they have no competing interests.

\section{Authors' contributions}

$\mathrm{HL}$ and MWC conceived the project, designed the experiments, and wrote the manuscript. $\mathrm{HL}$ and $\mathrm{BC}$ conducted the experiments. $\mathrm{HL}, \mathrm{BC}, \mathrm{AK}$ and $\mathrm{J}$ analyzed the data. MWC supervised the project. All authors read and approved the final manuscript.

\section{Acknowledgement}

This study was financially supported by the Competitive Research Program of the National Research Foundation of Singapore (NRF-CRP5-2009-03).

Received: 3 January 2013 Accepted: 19 June 2013

Published: 5 July 2013

\section{References}

1. Fischer CR, Klein-Marcuschamer D, Stephanopoulos G: Selection and optimization of microbial hosts for biofuels production. Metab Eng 2008, 10(6):295-304

2. Fortman JL, Chhabra S, Mukhopadhyay A, Chou H, Lee TS, Steen E, Keasling JD: Biofuel alternatives to ethanol: pumping the microbial well. Trends Biotechnol 2008, 26(7):375-381.

3. Lee SK, Chou H, Ham TS, Lee TS, Keasling JD: Metabolic engineering of microorganisms for biofuels production: from bugs to synthetic biology to fuels. Curr Opin Biotechnol 2008, 19(6):556-563.

4. Dunlop MJ: Engineering microbes for tolerance to next-generation biofuels. Biotechnol Biofuels 2011, 4:32.

5. Schirmer A, Rude MA, Li X, Popova E, del Cardayre SB: Microbial biosynthesis of alkanes. Science 2010, 329(5991):559-562.

6. Bernard A, Domergue F, Pascal S, Jetter R, Renne C, Faure JD, Haslam RP, Napier JA, Lessire R, Joubes J: Reconstitution of plant alkane biosynthesis in yeast demonstrates that Arabidopsis ECERIFERUM1 and ECERIFERUM3 are core components of a very-long-chain alkane synthesis complex. Plant Cell 2012, 24(7):3106-3118.

7. Pinkart HC, White DC: Phospholipid biosynthesis and solvent tolerance in Pseudomonas putida strains. J Bacteriol 1997, 179(13):4219-4226.

8. Sikkema J, de Bont JA, Poolman B: Mechanisms of membrane toxicity of hydrocarbons. Microbiol Rev 1995, 59(2):201-222.

9. Nuwaysir EF, Bittner M, Trent J, Barrett JC, Afshari CA: Microarrays and toxicology: the advent of toxicogenomics. Mol Carcinog 1999, 24(3):153-159.

10. Afshari CA, Nuwaysir EF, Barrett JC: Application of complementary DNA microarray technology to carcinogen identification, toxicology, and drug safety evaluation. Cancer Res 1999, 59(19):4759-4760.

11. Chang W, Toghrol F, Bentley WE: Toxicogenomic response of Staphylococcus aureus to peracetic acid. Environ Sci Technol 2006, 40(16):5124-5131.

12. Carvalho RN, Bopp SK, Lettieri T: Transcriptomics responses in marine diatom Thalassiosira pseudonana exposed to the polycyclic aromatic hydrocarbon benzo[a]pyrene. PLoS One 2011, 6(11):e26985. 
13. Kang A, Chang MW: Identification and reconstitution of genetic regulatory networks for improved microbial tolerance to isooctane. Mol Biosyst 2012, 8(4):1350-1358.

14. Matsui K, Hirayama T, Kuroda K, Shirahige K, Ashikari T, Ueda M: Screening for candidate genes involved in tolerance to organic solvents in yeast. Appl Microbiol Biotechnol 2006, 71(1):75-79.

15. Ghosh AK, Ramakrishnan G, Rajasekharan R: YLR099C (ICT1) encodes a soluble Acyl-CoA-dependent lysophosphatidic acid acyltransferase responsible for enhanced phospholipid synthesis on organic solvent stress in Saccharomyces cerevisiae. J Biol Chem 2008, 283(15):9768-9775.

16. Dos Santos SC, Teixeira MC, Cabrito TR, Sa-Correia I: Yeast toxicogenomics: genome-wide responses to chemical stresses with impact in environmental health, pharmacology, and biotechnology. Front Genet 2012, 3:63.

17. Yasokawa D, Murata S, Kitagawa E, Iwahashi Y, Nakagawa R, Hashido T, Iwahashi H: Mechanisms of copper toxicity in Saccharomyces cerevisiae determined by microarray analysis. Environ Toxicol 2008, 23(5):599-606.

18. Yasokawa D, Iwahashi H: Toxicogenomics using yeast DNA microarrays. J Biosci Bioeng 2010, 110(5):511-522.

19. Harger M, Zheng L, Moon A, Ager C, An JH, Choe C, Lai Y-L, Mo B, Zong D, Smith MD, et al: Expanding the product profile of a microbial alkane biosynthetic pathway. ACS Synth Biol 2012, 2(1):59-62.

20. de Smet MJ, Kingma J, Witholt B: The effect of toluene on the structure and permeability of the outer and cytoplasmic membranes of Escherichia coli. Biochim Biophys Acta 1978, 506(1):64-80.

21. Inoue A, Horikoshi K: A pseudomonas thrives in high-concentrations of toluene. Nature 1989, 338(6212):264-266.

22. Kawamoto T, Kanda T, Tanaka A: Preparation of an organic solventtolerant strain from baker's yeast. Appl Microbiol Biotechnol 2001, 55(4):476-479.

23. Gill CO, Ratledge C: Toxicity of n-Alkanes, n-Alk-1-enes, n-Alkan-1-ols and n-Alkyl-1-bromides towards Yeasts. Microbiology 1972, 72(1):165-172.

24. Mewes HW, Frishman D, Guldener U, Mannhaupt G, Mayer K, Mokrejs M, Morgenstern B, Munsterkotter M, Rudd S, Weil B: MIPS: a database for genomes and protein sequences. Nucleic Acids Res 2002, 30(1):31-34.

25. Bissinger $\mathrm{PH}, \mathrm{Kuchler} \mathrm{K}$ : Molecular cloning and expression of the Saccharomyces cerevisiae STS1 gene product. A yeast ABC transporter conferring mycotoxin resistance. J Biol Chem 1994, 269(6):4180-4186.

26. Grigoras I, Lazard M, Plateau P, Blanquet S: Functional characterization of the Saccharomyces cerevisiae $A B C$-transporter Yor1 $p$ overexpressed in plasma membranes. Biochim Biophys Acta 2008, 1778(1):68-78.

27. Kolaczkowski M, Kolaczowska A, Luczynski J, Witek S, Goffeau A: In vivo characterization of the drug resistance profile of the major $A B C$ transporters and other components of the yeast pleiotropic drug resistance network. Microb Drug Resist 1998, 4(3):143-158.

28. Mahe Y, Parle-McDermott A, Nourani A, Delahodde A, Lamprecht A, Kuchler K: The ATP-binding cassette multidrug transporter Snq2 of Saccharomyces cerevisiae: a novel target for the transcription factors Pdr1 and Pdr3. Mol Microbiol 1996, 20(1):109-117.

29. Ernst R, Klemm R, Schmitt L, Kuchler K: Yeast ATP-binding cassette transporters: cellular cleaning pumps. Methods Enzymol 2005, 400:460-484.

30. Garay-Arroyo A, Covarrubias AA: Three genes whose expression is induced by stress in Saccharomyces cerevisiae. Yeast 1999, 15(10A):879-892.

31. Schneiter R, Guerra CE, Lampl M, Tatzer V, Zellnig G, Klein HL, Kohlwein SD: A novel cold-sensitive allele of the rate-limiting enzyme of fatty acid synthesis, acetyl coenzyme A carboxylase, affects the morphology of the yeast vacuole through acylation of Vac8p. Mol Cell Biol 2000, 20(9):2984-2995.

32. Hong ME, Lee KS, Yu BJ, Sung YJ, Park SM, Koo HM, Kweon DH, Park JC, Jin YS: Identification of gene targets eliciting improved alcohol tolerance in Saccharomyces cerevisiae through inverse metabolic engineering. J Biotechnol 2010, 149(1-2):52-59.

33. Monteiro PT, Mendes ND, Teixeira MC, d'Orey S, Tenreiro S, Mira NP, Pais H, Francisco AP, Carvalho AM, Lourenco AB, et al: YEASTRACT-DISCOVERER: new tools to improve the analysis of transcriptional regulatory associations in Saccharomyces cerevisiae. Nucleic Acids Res 2008, 36(Database issue):D132-D136

34. Li XZ, Zhang L, Poole K: Role of the multidrug efflux systems of Pseudomonas aeruginosa in organic solvent tolerance. J Bacterio/ 1998, 180(11):2987-2991
35. Ramos JL, Duque E, Gallegos MT, Godoy P, Ramos-Gonzalez MI, Rojas A, Teran W, Segura A: Mechanisms of solvent tolerance in gram-negative bacteria. Annu Rev Microbiol 2002, 56:743-768

36. Rojas A, Duque E, Mosqueda G, Golden G, Hurtado A, Ramos JL, Segura A: Three efflux pumps are required to provide efficient tolerance to toluene in Pseudomonas putida DOT-T1E. J Bacteriol 2001, 183(13):3967-3973.

37. Tsukagoshi N, Aono R: Entry into and release of solvents by Escherichia coli in an organic-aqueous two-liquid-phase system and substrate specificity of the AcrAB-TolC solvent-extruding pump. J Bacterio/ 2000, 182(17):4803-4810.

38. Coleman JJ, Mylonakis E: Efflux in fungi: la piece de resistance. PLoS Pathog 2009, 5(6):e1000486.

39. Paumi CM, Chuk M, Snider J, Stagljar I, Michaelis S: ABC transporters in Saccharomyces cerevisiae and their interactors: new technology advances the biology of the ABCC (MRP) subfamily. Microbiol Mol Biol Rev 2009, 73(4):577-593.

40. Dunlop MJ, Dossani ZY, Szmidt HL, Chu HC, Lee TS, Keasling JD, Hadi MZ, Mukhopadhyay A: Engineering microbial biofuel tolerance and export using efflux pumps. Mol Syst Biol 2011, 7:487.

41. Koronakis V, Sharff A, Koronakis E, Luisi B, Hughes C: Crystal structure of the bacterial membrane protein TolC central to multidrug efflux and protein export. Nature 2000, 405(6789):914-919.

42. Li XZ, Nikaido H: Efflux-mediated drug resistance in bacteria: an update. Drugs 2009, 69(12):1555-1623.

43. Bokma E, Koronakis E, Lobedanz S, Hughes C, Koronakis V: Directed evolution of a bacterial efflux pump: adaptation of the E. coli TolC exit duct to the Pseudomonas MexAB translocase. FEBS Lett 2006, 580(22):5339-5343

44. Huffer S, Roche CM, Blanch HW, Clark DS: Escherichia coli for biofuel production: bridging the gap from promise to practice. Trends Biotechnol 2012, 30(10):538-545

45. Lo T-M, Teo WS, Ling $H$, Chen B, Kang A, Chang MW: Microbial engineering strategies to improve cell viability for biochemical production. Biotechnol Adv 2013. in press.

46. Maeng JH, Sakai Y, Tani Y, Kato N: Isolation and characterization of a novel oxygenase that catalyzes the first step of $n$-alkane oxidation in Acinetobacter sp. strain M-1. J Bacteriol 1996, 178(13):3695-3700.

47. Lee TI, Rinaldi NJ, Robert F, Odom DT, Bar-Joseph Z, Gerber GK, Hannett NM, Harbison CT, Thompson CM, Simon I, et al: Transcriptional regulatory networks in Saccharomyces cerevisiae. Science 2002, 298(5594):799-804.

48. Gueldener U, Heinisch J, Koehler GJ, Voss D, Hegemann JH: A second set of loxP marker cassettes for Cre-mediated multiple gene knockouts in budding yeast. Nucleic Acids Res 2002, 30(6):e23.

49. Manivasakam P, Schiestl RH: High efficiency transformation of Saccharomyces cerevisiae by electroporation. Nucleic Acids Res 1993, 21(18):4414-4415.

\section{doi:10.1186/1754-6834-6-95}

Cite this article as: Ling et al:: Transcriptome response to alkane biofuels in Saccharomyces cerevisiae: identification of efflux pumps involved in alkane tolerance. Biotechnology for Biofuels 2013 6:95.

\section{Submit your next manuscript to BioMed Central and take full advantage of:}

- Convenient online submission

- Thorough peer review

- No space constraints or color figure charges

- Immediate publication on acceptance

- Inclusion in PubMed, CAS, Scopus and Google Scholar

- Research which is freely available for redistribution 